\title{
Surviving stress during metastasis
}

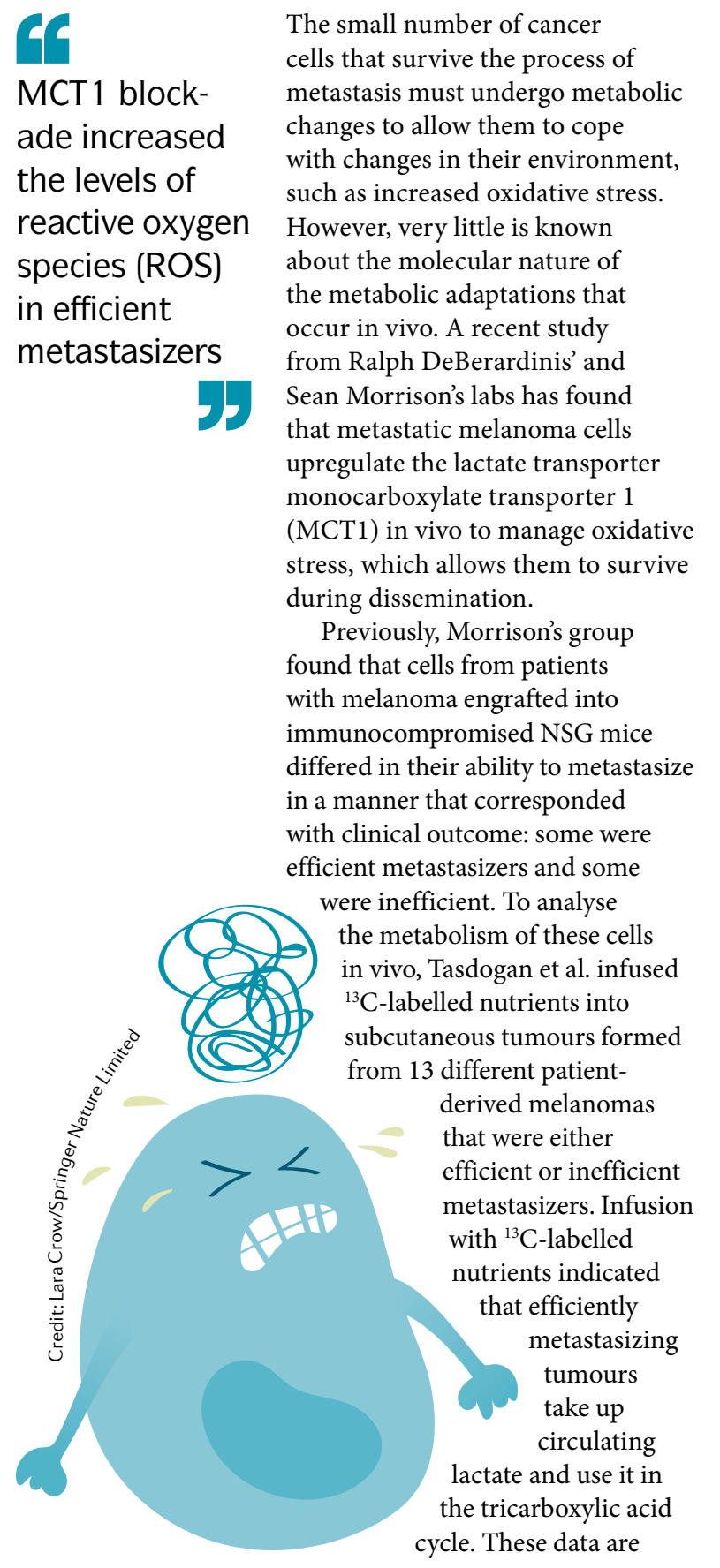

consistent with previous data from DeBerardinis' group showing that aggressive lung cancers use lactate as a fuel in vivo.

Immunofluorescence and flow cytometry studies then indicated that efficient metastasizers have increased levels of MCT1 on the cell surface. MCT1 is a bidirectional transporter of lactate and other monocarboxylates, but the main physiological role of MCT1 is lactate import. Indeed, the authors found that treating three different efficiently metastasizing melanomas in NSG mice with the MCT1-selective inhibitor AZD3965 reduced lactate uptake. In addition, AZD3965 treatment of mice with established tumours, or knockdown of MCT1 using short hairpin RNA, reduced the number of circulating tumour cells as well as metastatic burden, with only minimal effect on primary tumour growth. Inhibition of MCT1 in an immunocompetent mouse model of melanoma with AZD3965 or CRISPR-mediated Mct1 knockout similarly reduced metastatic burden without affecting primary tumour growth.

Looking at levels of MCT1 expression on cells within the same mouse, the authors found that cells expressing high levels of MCT1 $\left(\mathrm{MCT}^{\text {high }}\right)$ were enriched in the blood compared with primary subcutaneous tumours. When MCT $1^{\text {high }}$ cells and cells with low MCT1 expression (MCT1 $1^{\text {low }}$ ) were isolated and re-implanted subcutaneously into mice, there was no difference in primary tumour growth. However, when they were injected intravenously,
MCT $1^{\text {high }}$ cells formed more metastatic tumours than did MCT $1^{\text {low }}$ cells, suggesting a differential ability of these cells to survive during metastasis.

To look more closely at cell survival, the authors resected the primary melanomas to extend mouse survival and treated the mice with AZD3965 either before or after resection. Mice treated before tumour resection had reduced metastatic burden, but there was no effect on mice treated after resection, suggesting that AZD3965 is acting on cells during their initial systemic dissemination as opposed to during growth at distant sites.

Molecularly, MCT1 blockade increased the levels of reactive oxygen species (ROS) in efficient metastasizers. Treatment with AZD3965 plus an antioxidant increased metastatic burden compared with AZD3965 alone, indicating that the inhibitory effect of AZD3965 on metastasis is at least partially owing to increased oxidative stress in these cells. The authors also observed reduced flux through the oxidative pentose phosphate pathway (PPP) in tumours from mice treated with AZD3965. The oxidative PPP produces NADPH, which is then used by cells to counteract ROS and decrease oxidative stress, so the reduced PPP flux is consistent with the increased ROS levels observed in AZD3965-treated melanomas. This reduction in flux through the PPP might be a result of the increased $\mathrm{pH}$ in cells following MCT1 inhibition (as lactate is co-transported with $\mathrm{H}^{+}$).

These data suggest that MCT1 could potentially be targeted to inhibit the survival of metastatic melanoma cells in the blood before they colonize distant sites.

Sarah Seton-Rogers

ORIGINAL ARTICLE Tasdogan, A. et al. Metabolic heterogeneity confers differences in melanoma metastatic potential. Nature 577, 115-120 (2020) 